\title{
RECOGNITION FOR THE BOUNDARY OF PRESSURE-TOLERANT AND PRESSURE-RELIEF AREAS ON THE SCANNED POINTS OF RESIDUAL LIMB
}

\author{
${ }^{2}$ LaI-Hsing Hsu, ${ }^{1}$ Hon-Wing Au, ${ }^{1}$ You-Li Chou AND ${ }^{1}$ Gwo-Feng Huang \\ ${ }^{1}$ Institute of Biomedical Engineering, and ${ }^{2}$ Department of Mechanical Engineering, \\ National Cheng Kung University, Tainan, TAIWAN
}

\begin{abstract}
An algorithm is proposed to recognize the pressure-relief area and pressure-tolerance area on the CAD (computer aided design)model of a residual limb that needs to be modified for the use of making socket. The recognition algorithm includes the basic techniques of image processing, geometric model transformation, and the characteristic distance between two adjacent scanned points. The results of the boundary points are transferred to a CAD system that is employed to modify the shape of the residual limb within these two types of areas according to the prosthetist requirements. The modified CAD model is then used to produce a better-fit socket.
\end{abstract}

Biomed Eng Appl Basis Comm, 2001 (December); 13: 276-282.

Keywords: pressure-tolerant area, pressure-relief area, $\mathrm{CAD}$ model, prosthetic socket, residual limb

\section{INTRODUCTION}

To accelerate the process of fitting and fabrication of artificial limbs, in the 1960s Foort [1] at the University of British Columbia proposed the use of numerical controlled milling machines based on the data supplied by stercophotography to produce a positive model of the stump that is ready for molding of a socket. Progress was slow not until the introduction of computeraided design techniques and personal computers. An automated process for molding polypropylene sockets, called Rapidform, has been developed in the University College London in the late 1970s. And, it seemed logical to develop a system that has involved computer-aided design, numerically controlled production of a positive model of the stump, and automated pro-

Received: Aug. 30, 2001; accepted: Oct. 12, 2001 Correspondance: You-Ii Chou, Professor Institute of Biomedical Engineering, National Cheng Kung University, 1 Ta-Hsueh Road, Tainan, Taiwan; E-mail: ylchou@mail.ncku.edu.tw duction of the socket. Researchers there also envisioned automated fabrication of the entire artificial limb if alignment data could be fed into the system.

In the early 1980 s at University College London, a series of steps has been introduced to duplicate the model of a residual limb. The system involves the following procedures: taking a loose cast of the transtibial amputation stump, transferring the inside contours to a personal computer by means of a digitizer to produce an image of a positive model, modifying the shape of the positive model on the screen, and transferring the information into a numerically controlled milling machine to carve a positive model from a blank of plaster of paris or wax. At this stage the positive model is ready for the use of fabricating a socket by vacuum forming method of polypropylene or similar sheet plastic.

In the mid-1980s the Veterans Administration began funding several projects in the United States to enhance the application of the CAD/CAM process in the fabrication of artificial limbs [2]. Although the interests generated by these projects, not a great deal of progress is apparent. Undoubtedly, further development will make $\mathrm{CAD} / \mathrm{CAM}$ a useful tool to improve 
service to amputees.

\section{PRESSURE-TOLERANT AND PRESSURE-RELIEF AREAS}

Pressure-tolerant (PT) area is the area where can support the load and pressure produced while the amputee walking or standing and pressure-relief (PR) area at where loading had better not exert otherwise injure will be caused. Figure 1 shows the PT and PR areas on residual limb.

The beginning of the process of fabricating prosthetic socket based on the technique of reverse engineering is digitizing the shape of residual limb, i.e. measured with laser scanner. After the $\mathrm{CAD}$ surface model is constructed, the anatomical areas on the CAD model of the residual limb have been modified, a positive model of the residual limb can be duplicated, and the socket for the specific residual limb can then be made [2]. The comfortability of prosthesis is mainly depending on the fitness of socket [3]. A better-fit socket represents that contact pressure on pressuretolerant and pressure-relief areas have been well distributed. Therefore, identification of PT and PR areas is an important process before modifying the shape of residual limb model within CAD system.

For an experienced prosthetist, it is easy to clearly mark PT and PR areas on residual limb of an amputee. There were many researches on defining the PT and PR areas using the facilities of ultrasound [4], MRI [5] or CT [6] to scan the inside structure of residual limb and then identify the modification areas. The result showed that there is no significant difference on the areas either marked by prosthesist or identified by using appropriate equipment. However, it is difficult to automatically identify those areas on computer images because residual limb is smooth and no obvious boundaries.

Improvement in accuracy and portability of laser scanner make amputees can be scanned more conveniently and with reasonable cost. However, as discussed

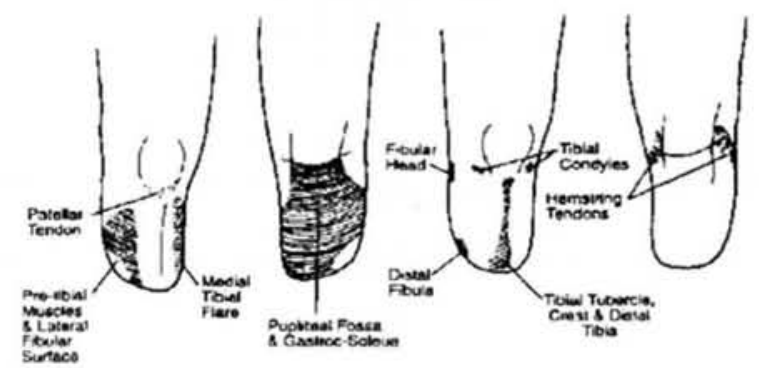

(a) PT areas

(b) PR areas

Fig. 1 The location of pressure-tolerant and pressure-relief areas on below-knee limb[3] previously the shortcoming of laser scanning is unable to obtain the inside structure of residual limb. Integration of the traditional area marking method and the digitized information generated from laser scanner, pressure-tolerant and pressure-relief areas can be identified from laser scanning data. The purpose of this study is to develop an algorithm for the recognition of boundary points between PT and PR areas.

When design the shape of socket, residual limb must contact only on pressure-tolerant area where can support the weight of amputee while standing and walking, but cannot contact the pressure-relief area, such as the bone of residual limb, where cannot support the weight. The rule of thumb for the prosthetic modification of the shape of residual limb plastic model includes: add plastic on the pressure-relief areas; remove plastic on the pressure-tolerant areas of duplicated positive model of residual limb. Wrap with PE on the modified plastic model after modification has been made and a better-fit socket can be provided to the amputee.

\section{RELATED PRINCIPLES}

\subsection{Projection of an Object}

Projecting an entity onto a plane or a surface may provide useful information for the applications such as determining shadows or finding the position of the entity relative to the plane or the surface. Point $P_{0}$ is projected along the direction onto a given plane. It is desired to calculate the coordinates of the projected point $\mathrm{Q}$. The plane equation can be written as follows:

$$
\vec{P}(u, v)-\vec{a}+u \vec{b}+v \vec{c}
$$

where $\vec{a}$ is a position vector and $\vec{b}$ and $\vec{c}$ are unit vectors as shown in Fig. 2 and $u$ and $v$ are scalar sizes along corresponding coordinate axes. The equation of the projection line is given by

$$
\vec{P}(w)=\vec{P}_{o}+w \vec{r}
$$

where $c$ and $w$ are the unit vector and scalar size

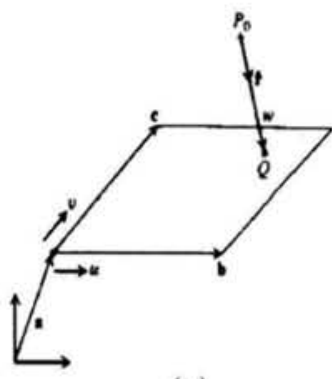

(a)

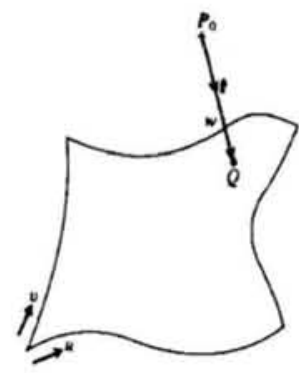

(b)
Fig. 2 Points projected onto surfaces 
along projection direction. The point $\mathrm{Q}$ is the intersection point between the projection line and the plane; that is, the following equation must be solved for $u, v$, and $\mathrm{w}$ :

$$
\begin{aligned}
& \vec{P}(u, v)-\vec{P}(w)=0 \\
& \dot{a}+u \vec{b}+v \vec{c}=\vec{P}_{0}+w \vec{r}
\end{aligned}
$$

To solve for w, dot-multiply both sides of the above equation by $(\vec{b} \times \vec{c})$ to get

$$
(\vec{b} \times \vec{c}) \cdot \vec{a}=(\vec{b} \times \vec{c}) \cdot\left(\vec{P}_{0}+w \vec{r}\right)
$$

since $(b \times c)$ is perpendicular to both $\mathrm{b}$ and $c$. Equation $(6)$ gives

$$
w=\frac{(\vec{b} \times \vec{c}) \cdot\left(\vec{a}-\vec{P}_{o}\right)}{(\vec{b} \times \vec{c}) \cdot \vec{r}}
$$

Similarly, we can write

$$
\begin{aligned}
u & =\frac{(\vec{c} \times \vec{r}) \cdot\left(\vec{P}_{o}-\vec{a}\right)}{(\vec{c} \times \vec{r}) \cdot \vec{b}} \\
\nu & =\frac{(\vec{b} \times \vec{r}) \cdot(\ddot{P}-\vec{a})}{(\vec{b} \times \vec{r}) \cdot \dot{c}}
\end{aligned}
$$

Substituting Eq. (5) into Eq. (2), or combining

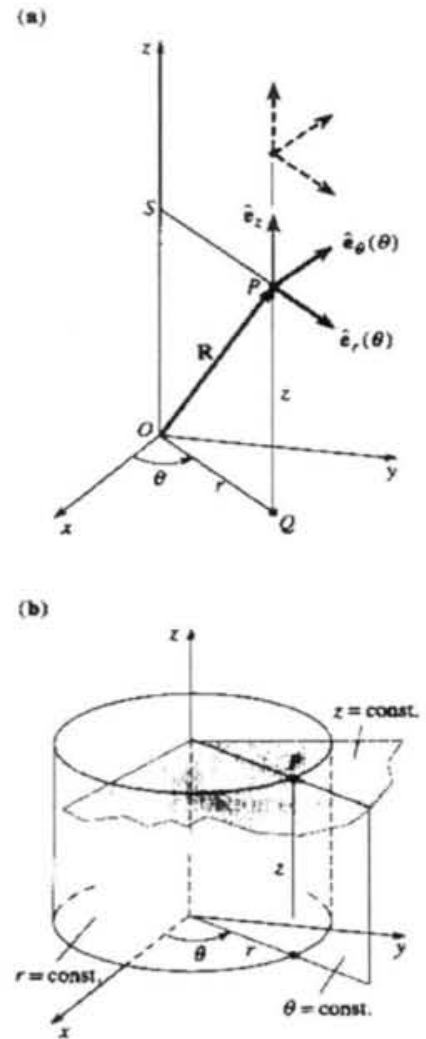

Fig. 3 Cylindrical coordinates
Eqs. (1), (7) and (8), can solve the projection point Q. If point $P_{0}$ is to be projected onto a general surface, such as cylindrical surface, $\mathrm{Eq}(1)$ is replaced by the appropriate surface equation. The cylindrical surface equation is denoted as followed

$$
C(u, v)=R^{2}-\left(u^{2}+v^{2}\right)
$$

And equation (3) becomes nonlinear equations.

\subsection{Cylindrical Coordinate System}

In a cylindrical coordinate system, $\mathrm{r}, \theta, \mathrm{z}$ are used to represent the coordinates for a point as shown in Fig. 3, and the variable $z$ is the same as that in Cartesian coordinate system. The original scanned points are denoted as Cartesian coordinates and can be transferred into cylindrical coordinates by the following equations before projection:

$$
\begin{aligned}
& r-\sqrt{X^{2}+Y^{2}} \\
& \vec{\theta}-\tan ^{-1}(Y \mid X)
\end{aligned}
$$

Since the shape of a below-knce residual limb is similar to a cylindrical surface, cylindrical coordinate system is suitably used to implement the algorithm in this study.

\subsection{The Process of Reverse Engineering}

The development of CAD/CAM technology has

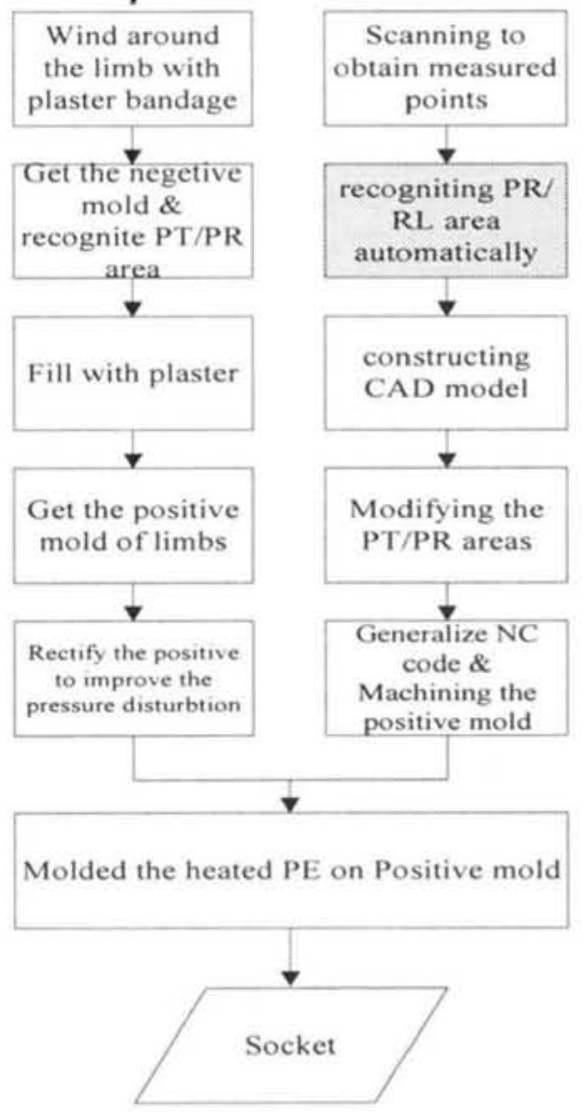

Fig. 4 The comparison of socket fabrication between traditional and CAD/CAM methods 


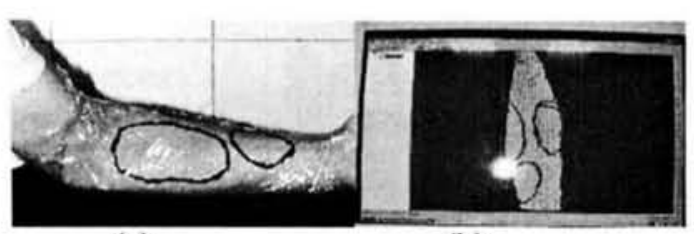

(a)

(b)

Fig. 5 The scanning effect with plastic wrap

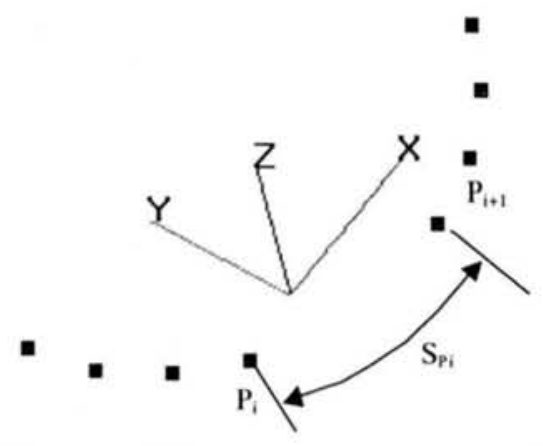

\section{Fig. 6 Distance between boundary points}

production. That increases the varieties of product. During Product development many CAD models are extracted from the existing models or aesthetic models directly, such as car model. Because the shapes of residual limbs are hardly defined by usual process of geometrical description, the technology of reverse engineering is therefore employed.

The process of reverse engineering [7] includes the following steps. Firstly, the shape of an existing model is measured by a non-intrusive scanning machine, such as CMM or laser scanner. Secondly, the scanned points should be properly selected and the different regions with specific characteristics had better be subdivided and are segmented. Thirdly, a surface model is then reconstructed using the segmented scanning points. Fourthly, the shape of the constructed CAD surface model may be modified according to the design requirement. Finally, the modified CAD model can then be used for production by $\mathrm{CNC}$ machines or the model can be used for engincering analysis, such as stress distribution by FEM. Residual limb cannot defined by geometry and reverse engineering is applied to setup the CAD model. The comparison of socket fabrication between traditional method and the use of reverse engineering is shown in Fig. 4.

\subsection{Recognition Method}

The basic elements of triangulation laser scanner is partial or directed illumination and a one- or twodimensional image converter which determines the position of the illuminated scene elements lying in its plane from a direction differing from that of illumination. From this information and from the known angle between the directions for illumination and the directions of observation, the spatial coordinates of the surface may be calculated. The illumination is not good on black regions and there will not produce any data points on these regions. The PT and PR areas must be clearly marked on the residual limb with black marker pen before laser scanning.

The Epithelium layer of skin is not smooth [8] that will influence the scanning effects. To prevent the skin from hurting by oily marker pen and whiteboard marker pen is used. The whiteboard marker pen will not stain the skin deeply and the scanning effect is not obvious. The solution is wrapping the residual limb with plastic wrap to smooth the surface and to protect the skin from hurt by oily marker pen that can stain deeply. The scanning result clearly shows the boundaries that are marked by experienced prosthetist as shown in Fig. 5.

After the sections selected, the distance between each two adjacent points on a same section is calculated and the boundary points are recognized. The criterion distance of two adjacent boundary points is the width of marker pen. The distance will be enlarged after projected onto a cylindrical surface and it will be proportional to the diameter of the cylinder. If the arc length $\widehat{S}_{p_{i}}$ (as shown in Fig. 6) is greater then criterion distance, these two adjacent points would be recognized as boundary points (i.e. $\mathrm{P}_{\mathrm{i}}$ and $\mathrm{P}_{\mathrm{i}+1}$ in Fig. 6). To prevent the influence of data point shortage (such as distal residual limb), the boundary points will be ignored if $\widehat{S}_{P i}$ is too large.

\section{A CASE STUDY TO IMPLEMENT THE RECOGNITION ALGORITHM}

The flow diagram of the proposed algorithm is depicted in Fig. 7. A duplicated cast model of a residual limb shown in Fig. 8 is used the case study to implement the recognition process. After the PT and PR areas are marked, the measured points shown in Fig. 9 are obtained by using a laser scanner. The scanned points are projected onto a cylindrical surface its radius is determined by the minimum size of a cylinder that cnables to enclose the whole scanned points. A value of $50 \mathrm{~mm}$ is used in this study. Figure 10 shows the projection result.

As the process presented in Fig. 7, a number of sections that is perpendicular to the $z$-axis are designated to catch the scanned points that are close to the sections within a certain distance, $0.1 \mathrm{~mm}$ in this study. The collected scanned points are shown in Fig. 10 after this procedure. The arc lengths between all two adjacent points on each same section are calculated. If the value of each arc length is larger than the criterion dis- 


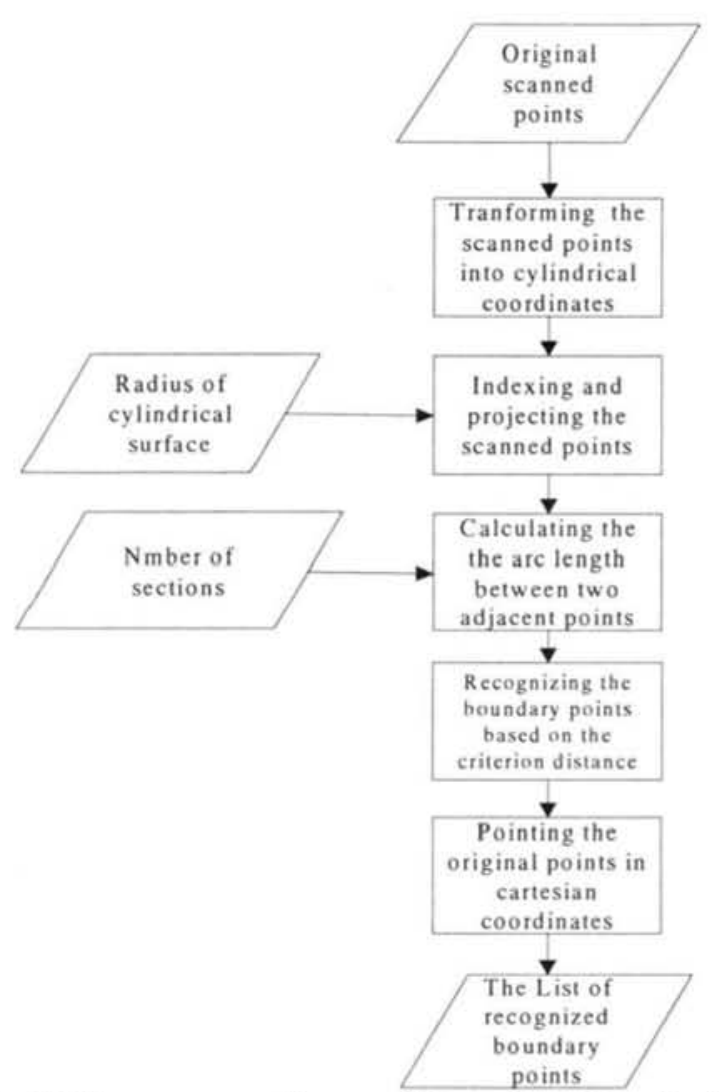

Fig. 7 The process of recognition algorithm for PT and $P R$ areas

tance, $3 \mathrm{~mm}$ designated in this study, these two adjacent points are then recognized as the boundary points. All the points on each section have been processed and all the boundary points represented in original Cartesian coordinates are kept in a list for the use of following process. Figure 12 shows the boundary points of all PT and PR areas of the case study.

\section{CONCLUSIONS AND DISCUS- SIONS}

The purpose of this study is to recognize the boundaries of pressure-relief and pressure-tolerance areas on residual limbs. The result of a case study has clcarly shown those areas on the CAD model that allows the prosthetist making the appropriate modification of the shape of the specific residual limb more easily and accurately. The conclusions of this study and recommendations for the future work are summarized as follows:

(1) Based on the expertise of prosthetist, clear marks of PT and PR areas on the residual limb can be recognized using the proposed algorithm and the scanned points of the marked residual limb.

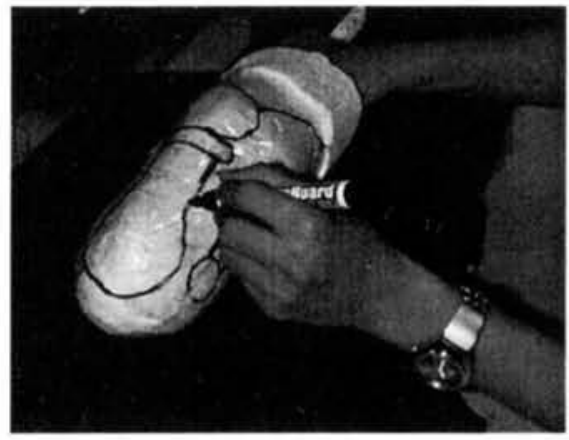

IFi.g. 8 Marking the PT and PR areas on the case :tudy

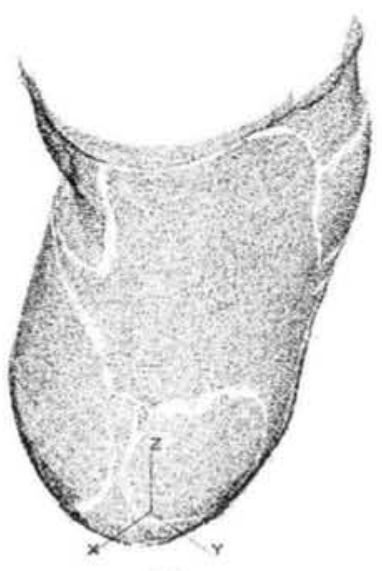

(a)

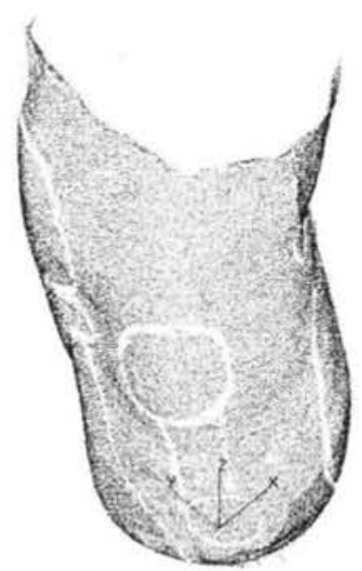

(b)
Fig. 9 The scanned points

(2) Only two values, i.e. the radius of projection cylinder and number of sections for segmentation, are required based on the proposed algorithm so that the boundary points of pressuretolerant area and pressure-rclief area can be recognized.

(3) The shortcoming of laser scanning that cannot view the inside structure of residual limb, such as bone, can be compensated by recognizing the marked boundarics of PT and PR areas.

(4) The scanned points of the recognized boundaries can be used for modifying the shapes of the measured limb so that a better-fit socket can be made based on the modified CAD model.

(5) A user-friendly system can be developed for the use of design prosthetic socket if more effort is arranged to integrate the proposed algorithm using the $\mathrm{CAD}$ techniques of surface reconstruction and modification.

(6) The result has shown that if the marked boundary is perpendicular to $z$-axis, less boundary points can be founded than that of the bound- 

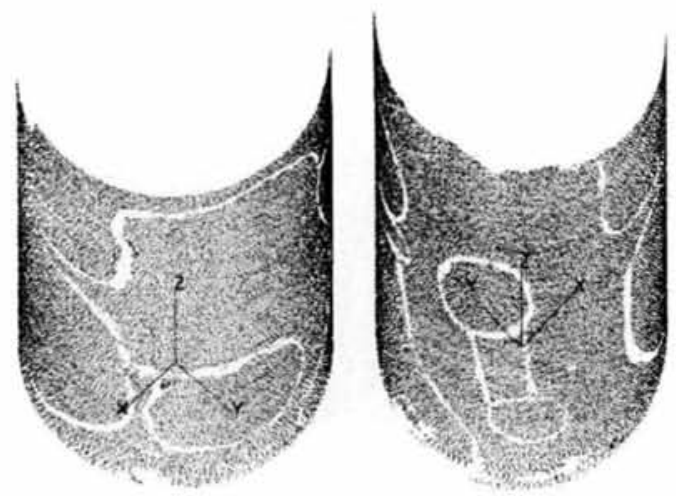

Fig. 10. Projection of scanning data onto the cylindrical surface (front and back sides)
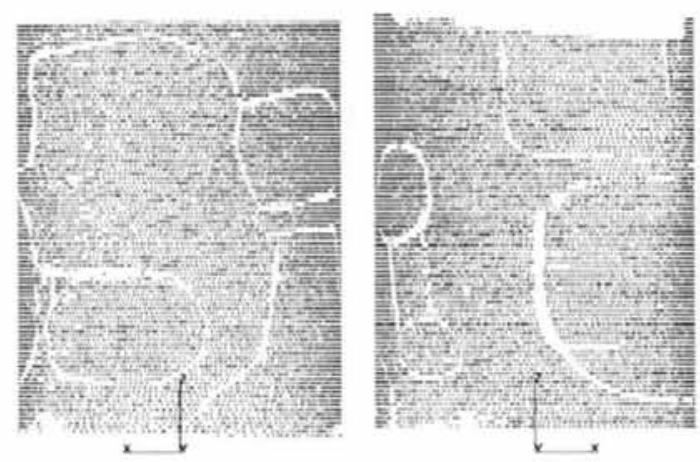

Fig. 11 Subdivided sections of scanned data (front and back sides)

ary parallel to $\mathrm{z}$-axis as shown in Fig. 13. The reason is that the direction of subdivided sections is perpendicular to $\mathrm{z}$-axis and more scanned points on one specific boundary within a specified region, $+/-0.1 \mathrm{~mm}$ of the $\mathrm{z}$ coordinate for each section in this study, will be projected onto the same level of section. However only two adjacent points can be recognized as boundary points for each specific boundary on one section. The solution is increasing the number of subdivided sections. Although the number of that boundary points projected onto the same section will be decreased, more number of subdivided sections can collect more boundary points. The number of boundary points being recognized will be increased. However, the number of points on each section will be too little if too many sections are divided and that will also influence the result of recognition. This is a trade-off problem. In the case study 100 sections are designated for the $150 \mathrm{~mm}$-length residual limb in this study.

(7) Only below-knee residual limb is implemented as case study in this study. Other residual

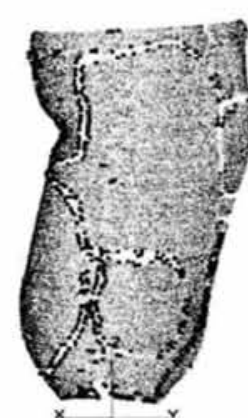

(a) front side

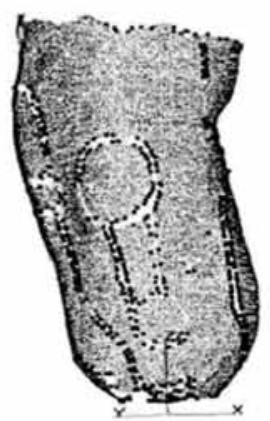

(b) back side
Fig. 12 Recognized boundary points (dots)

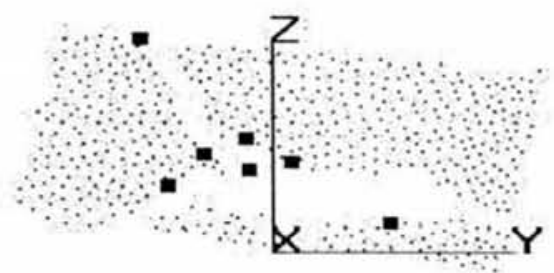

(a) 50 sections

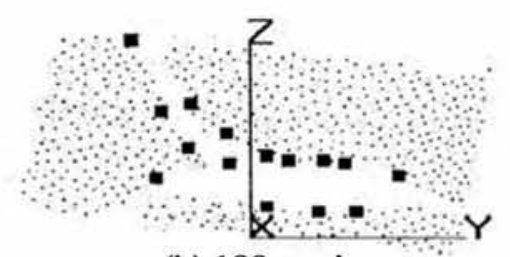

(b) 100 sections

Fig. 13 The results of different number of designated sections

limbs, such as above-knee residual limb, further effort is required to verify the proposed algorithm.

(8) As scanned points are enormous and irregular, data filtering is necessary. Space-sampling [7] is employed in this study to eliminate the points if the distance between two points is smaller than pre-set tolerance. This filtering method can reduce the sorting time while preprocessing of scanned points.

\section{ACKNOWLEDGEMENTS}

This research was supported by the National Health Research Institute (project no. NHRI-EX90$8817 \mathrm{EP})$. The use of a laser scanning machine in the Department of Industry Design at National Cheng Kuang University and the kind assistance from Profes- 
sor S. W. Hsiao and Mr H. T. Tsai are highly appreciated.

\section{REFERENCES}

1. Wilson A B. History of amputation surgery and prosthetics. In: Bowker JH, Michael JW, Eds. Atlas of Limb Prosthetics: Surgical, Prosthetic, and Rehabilitation Principles. Mosby-Year Book. 1992; 316.

2. Walsh NE, Lancaster JL, Faulkner VW and Rogers WE: A computerized system to manufacture prostheses for amputees in developing countries. Journal of Prosthetics and Orthotics, 1997; 1(3): 165181.

3. Engstrom B and Van de Ven C: Physiotherapy for amputees, Churchill Livingstone, 1985.

4. Ping He and Kefu Xue: A PC-based ultrasonic data acquisition system for computer-aided prosthetic socket design. IEEE transactions on rehabilitation engineering 1996; 4(2): 114-119.

5. Zhang M, Mark A F. T., and Chung A I. K: MRI investigation of musculoskeletal action of transfemoral residual limb inside a prosthetic socket. Medicine and Biology Society, 1998; 20(5): 274143.

6. DeMeis R: Body scanners give prostheses perfect fit. Design News. Boston, 1998.2.

7. Varay T., Martin RR, and Coxt, J: Reverse engineering of geometric models - an introduction. Computer Aided Design. 1997;29(4):255-268.

8. Marieb EN, and Mallatt J, Human Anatomy, Benjamin/Cummings, 1996. 